\title{
Partially integrated cantilever-based airborne nanoparticle detector for continuous carbon aerosol mass concentration monitoring
}

\author{
H. S. Wasisto ${ }^{1,2}$, S. Merzsch ${ }^{1, *}$, E. Uhde ${ }^{3}$, A. Waag ${ }^{1,2}$, and E. Peiner ${ }^{1,2}$ \\ ${ }^{1}$ Institut für Halbleitertechnik (IHT), Technische Universität Braunschweig, Braunschweig, Germany \\ ${ }^{2}$ Laboratory for Emerging Nanometrology (LENA), Braunschweig, Germany \\ ${ }^{3}$ Material Analysis and Indoor Chemistry Department (MAIC), Fraunhofer-WKI, Braunschweig, Germany \\ *now at: Infineon Technologies AG, Munich, Germany
}

Correspondence to: H. S. Wasisto (h.wasisto@tu-bs.de)

Received: 27 November 2014 - Revised: 19 February 2015 - Accepted: 19 February 2015 - Published: 11 March 2015

\begin{abstract}
The performance of a low-cost partially integrated cantilever-based airborne nanoparticle (NP) detector (CANTOR-1) is evaluated in terms of its real-time measurement and robustness. The device is used for direct reading of exposure to airborne carbon engineered nanoparticles (ENPs) in indoor workplaces. As the main components, a miniaturized electrostatic aerosol sampler and a piezoresistive resonant silicon cantilever mass sensor are employed to collect the ENPs from the air stream to the cantilever surfaces and to measure their mass concentration, respectively. Moreover, to realize a real-time measurement, a frequency tracking system based on a phase-locked loop (PLL) is built and integrated into the device. Long-term ENP exposure and a wet ultrasonic cleaning method are demonstrated to estimate the limitation and extend the operating lifetime of the developed device, respectively. By means of the device calibrations performed with a standard ENP monitoring instrument of a fast mobility particle sizer (FMPS, TSI 3091), a measurement precision of ENP mass concentrations of $<55 \%$ and a limit of detection (LOD) of $<25 \mu \mathrm{g} \mathrm{m}^{-3}$ are obtained.
\end{abstract}

\section{Introduction}

Over the last few decades, nanotechnology, which covers a compilation of technologies and methods for manipulating material on the nanoscale (i.e., nanomaterial or nanoparticle (NP)), has been attracting immense attention in society and has been hailed by some scientists as the next industrial revolution. The possible interests in nanotechnology originate mainly from the novel properties and characteristics of the nanomaterials, which are not the same as bulk materials and may be unpredictable and unimagined as scale effects (Maynard, 2007; Hullman, 2007). Thus, this technology has rapidly been developed and used across a variety of industries (e.g., electronics, medicine, cosmetics, pharmaceuticals, food packaging, household appliances, and national defense), leading to increased economic growth and new job vacancies (Bekker et al., 2013).
Although nanotechnology provides society with enormous feasibilities, questions have also been raised about uncertainties concerning the risks to and potential health effects of released NPs on the environment. The formation and release of the NPs into indoor environments and workplaces can occur through both incidental (i.e., unintentional NPs) and planned manufacturing processes (i.e., engineered NPs (ENPs)) (Balbus et al., 2007). Moreover, much higher awareness should be given to the workers, who manufacture and handle NPs directly in large quantities during the line productions (Brouwer, 2010). Thus, a direct-reading airborne NP mass concentration detector is very useful for the assessment of personal- and location-dependent monitoring in workplaces and indoor environments.

For individual NP mass monitoring, the complete system relies on mini portable devices that can be held and carried easily by the workers. Currently, the already developed NP mass sensors based on microelectromechanical systems 
(MEMS) need to be operated at high flow velocity (50$150 \mathrm{~m} \mathrm{~s}^{-1}$ ) using an external pump (Schmid et al., 2013) or partial vacuum requiring bulky, heavy, and expensive vacuum tools (Hajjam et al., 2011). In contrast, our first generation of the cantilever-based airborne NP detector (CANTOR1) evaluated in this work has a small size, a low weight, an appropriate flow rate, an atmospheric pressure working condition, and low-cost components. This device had been successfully tested to prove the NP mass sensing principle with detected ENPs down to $\sim 20 \mathrm{~nm}$ in diameter (Wasisto et al., 2013a). However, these measurements were done offline, where the ENP sampling and the sensor characterization were performed separately and controlled manually. Thus, some supporting components were needed to be added to the device. Recently, we reported on a frequency tracking system based on a phase-locked loop (PLL) circuit and a high voltage (HV) module based on a DC amplifier for nanoparticle mass concentration monitoring in real time (Wasisto et al., 2014). This new device (CANTOR-1) was successfully tested in normal indoor ambience, showing the ability to detect, e.g., cigarette smoke. However, the quantitative detection of NPs was impeded by coarse particulate matter, which was also deposited on the cantilever. To ensure that CANTOR-1 monitors only NPs, coarse particles have to be removed from the air passing through the NP sampler. Furthermore, to give a quantitative NP concentration read-out, CANTOR-1 had to be calibrated under defined ambient conditions.

Therefore, in this paper, we address a first quantitative test and evaluation of CANTOR-1 in real-time ENP measurements. For this purpose, measurements were performed under a defined ENP exposition in a sealed chamber and with standard stationary aerosol monitoring equipment; i.e., a fast mobility particle sizer (FMPS) was used to provide reference data for the time-dependent ENP concentration. Membrane filters and impactors were described to prevent interfering coarse particulate matter from entering the sampler. A recycling process of ultrasonic wet cleaning is also performed to regenerate the sensor after being fully loaded by ENPs during long-term exposure.

\section{Direct-reading cantilever-based airborne nanoparticle detector}

\subsection{Main components}

Figure 1a shows the principle of the direct-reading CANTOR-1. Particle-laden air is flown through a filter, which removes coarse particulate matter, towards a cantilever biased to a negative potential ( $\left.V_{\mathrm{EP}}\right)$ by which the positively charged fraction of the airborne nanoparticles is attracted. The mass added to the cantilever by the attached nanoparticles lowers the resonant frequency of the cantilever, which is driven by a piezostack $\left(V_{\mathrm{dr}}\right)$ and read-out $(\Delta V)$ using an integrated piezoresistive strain gauge supplied by $V_{0}$.
In principle, the CANTOR-1 system comprises two main components (i.e., a miniaturized electrostatic aerosol sampler and a piezoresistive resonant silicon cantilever mass sensor). The first main module is a cylindrical miniaturized electrostatic NP sampler made of aluminum material and designed as a tube with a diameter of $20 \mathrm{~mm}$ and a length of $45 \mathrm{~mm}$ (Fig. 1b). The tube consists of three single aluminum parts joined by threads denoted as inlet part, middle part, and outlet part, respectively. Aiming to direct the aerosol flow against the cantilever positioned on the tube axis, the inlet part was conically shaped with an inclination of $15^{\circ}$ to the tube axis (Fig. 1c). In the middle part of the tube, the second module of a silicon cantilever resonator was mounted by double-sided tape on a small ceramic printed circuit board (PCB) to integrate it with the piezoelectric stack actuator (Fig. 1d). Gold bonding microwires of $30 \mu \mathrm{m}$ were used for getting electrical contact between cantilever and board. To generate stable aerosol flow of $0.68 \mathrm{~L} \mathrm{~min}^{-1}$, a small fan (MF10A03A, SEPA Europe $\mathrm{GmbH}$ ) was mounted at the outlet part of the tube. To operate the NP sampler, a voltage of $3.5 \mathrm{~V}$ from either a battery pack or a power supply (HP E3631A) was used. This voltage, however, needed to be amplified first up to $0.5 \mathrm{kV}$ using a DC voltage amplifier (i.e., a factor of $\sim 140$ higher).

The employed second module is a silicon cantilever $\quad\left(V_{\text {cant }}=2750 \times 100 \times 50 \mu \mathrm{m}^{3}, \quad m_{\text {cant }}=32.04 \mu \mathrm{g}\right.$, $f_{\text {cant }}=9.4 \mathrm{kHz}$ ), which has a full square Wheatstone bridge on its clamped end as a piezoresistive element and works based on the strain-to-resistivity change to read the sensor signal output (Fig. 1e) (Wasisto et al., 2013b). The cantilevers are fabricated by utilizing silicon bulk micromachining processes (i.e., photolithography, thermal oxidation, dopant diffusion/implantation, and inductively coupled plasma (ICP) cryogenic deep reactive ion etching (cryo-DRIE)). Figure 2 depicts the fabrication process steps of the self-sensing piezoresistive silicon cantilever resonators, which can be described in detail as follows.

1. The fabrication was started with a preparation of (100)oriented $n$-type bulk silicon wafers $(3-5 \Omega \mathrm{cm})$ with a thickness of $300 \mu \mathrm{m}$. In contrast to piezoelectric AlN/Si cantilever resonators (Sökmen et al., 2010), which use SOI for the device fabrication, bulk silicon wafers are more preferable for the current sensor fabrication considering their lower price, high stability, high mechanical $Q$ factor, and high degree of freedom for the geometrical resonant cantilever design. At the beginning, a wafer was placed in a furnace to grow a thermal silicon dioxide $\left(\mathrm{SiO}_{2}\right)$ thin layer on the silicon surface. A $400 \mathrm{~nm} \mathrm{SiO} 2$ layer was obtained within $1 \mathrm{~h}$ at a temperature of $1100^{\circ} \mathrm{C}$.

2. Subsequently, the oxidized wafer coated with a photoresist mask was patterned utilizing UV lithography. Buffered hydrofluoric acid solution was then used to open the areas for the definition of $p$-type piezoresis- 


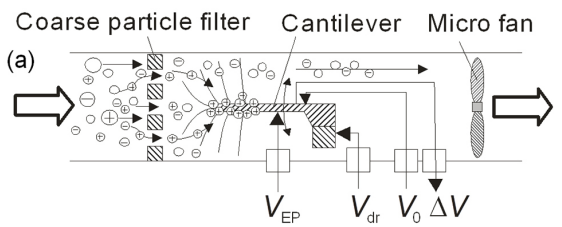

(b)
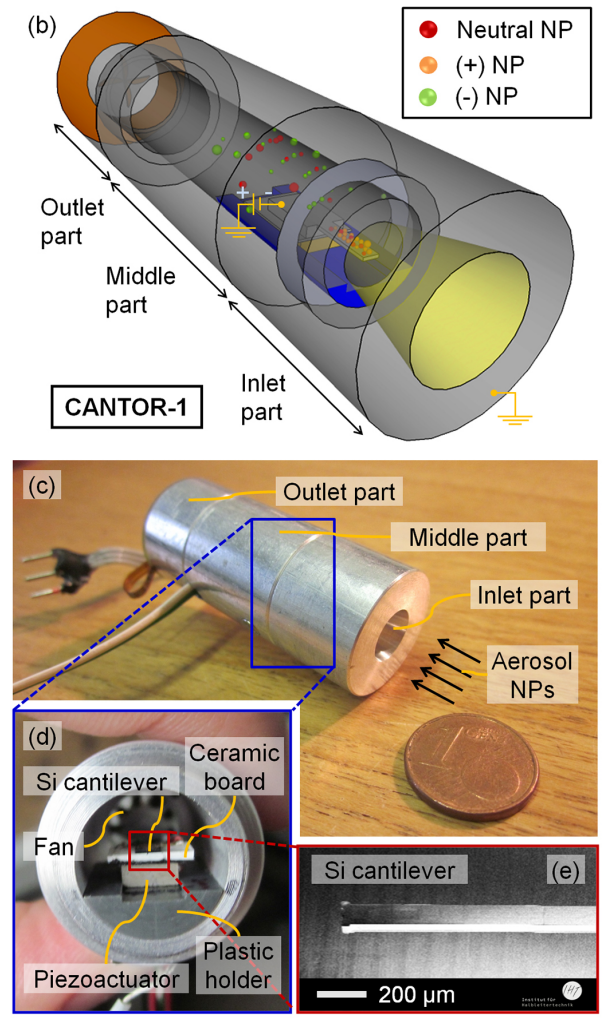

Figure 1. (a) 2-D and (b) 3-D schematics, and (c) photographs of the handheld CANTOR-1 showing (d) the main components of its middle part. (e) SEM image of the silicon piezoresistive cantilever resonator.

tors. Afterwards, to create a full piezoresistive Wheatstone bridge on the Si wafer, boron implantation or diffusion was performed.

3. Furthermore, to form $p^{+}$feed lines to the Wheatstone bridge and improve contact formation, additional boron diffusion or implantation was used. For the implanted and diffused wafers, the standard deviations of the measured resistivity from the desired value were $\sim 0.6$ and $\sim 4.1 \%$ in proportion to their lateral doping distribution, respectively. Typical values of the junction depth and the surface doping concentration amounted to $\sim 4.5 \mu \mathrm{m}$ and $1.5-3.0 \times 10^{18} \mathrm{~cm}^{-3}$, respectively, representing a tradeoff between a large piezoresistive coefficient $\pi_{44} \approx 1 \mathrm{GPa}^{-1}$ and a low temperature coefficient around $-3 \times 10^{-3}{ }^{\circ} \mathrm{C}^{-1}$ (Cho et al., 2008; Peiner et al., 2008).

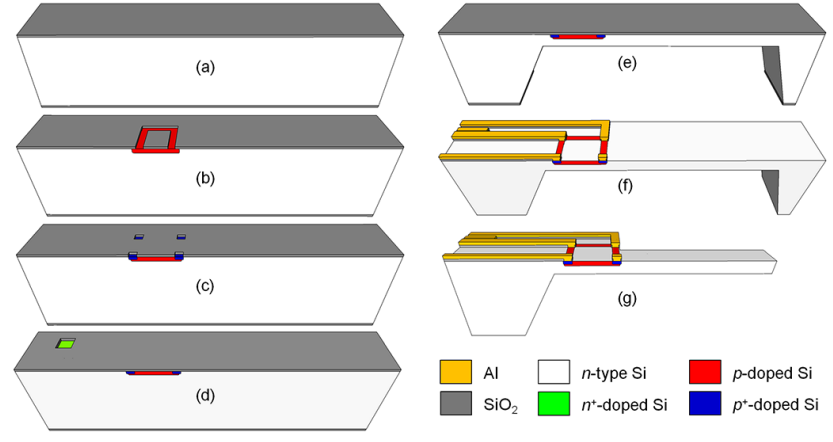

Figure 2. The fabrication process flow of the self-sensing piezoresistive silicon cantilever resonators showing (a) thermal oxidation, (b) $p$-type piezoresistor creation, (c) $p^{+}$-type contact formation, (d) phosphorus diffusion, (e) backside membrane wet etching, (f) metallization, and (g) cantilever free release dry etching.

4. Since an ohmic contact was needed for electrostatic NP collection, a phosphorus diffusion/implantation was then carried out and placed in an $n^{+}$-type well close to one of the $p^{+}$-type piezoresistor contacts.

5. Next, to create the backside membranes on the bottom side of the wafer, the selected bottom oxide layers were patterned by UV lithography and buffered Hydrofluoric acid (HF) etching. Through the oxide openings, a potassium hydroxide solution $(\mathrm{KOH})$ at elevated temperatures or cryo-DRIE was introduced to etch the silicon down to a residual thickness of $25-50 \mu \mathrm{m}$. This process is very critical because it determines the cantilever thickness and thus its operating resonant frequency. From the experiments, a tolerance of $\pm 0.5 \mu \mathrm{m}$ (i.e., 1 to $2 \%$ ) was normally obtained using $\mathrm{KOH}$ etching.

6. Subsequent to the opening of the $\mathrm{SiO}_{2}$ contact holes, a top-side metallization was then deposited by $300 \mathrm{~nm}$ aluminum electron-beam evaporation. Furthermore, to provide large-enough landing areas for non-permanent electrical connections using spring-loaded contact pins, large contact pads were provided (i.e., designed as a $0.75 \times 1 \mathrm{~mm}^{2}$ large area).

7. To finish the fabrication process, the cantilevers were lithographically patterned and released by cryo-DRIE from the front side of the samples. A dry etcher (SI 500 C, SENTECH Instruments GmbH, Berlin, Germany) was used along with a photoresist serving as the etching mask.

\subsection{Microparticle filtration components}

In addition, to filter out the undesired microparticles, which could possibly approach the cantilever, two filtration stages 


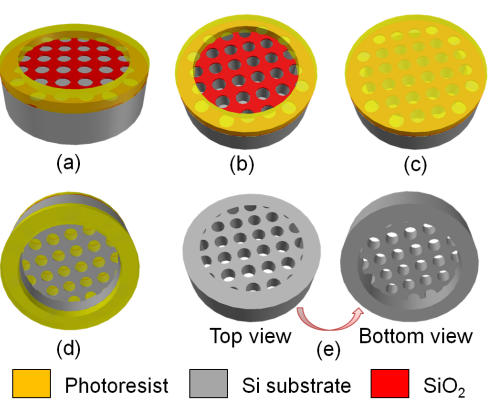

Figure 3. Fabrication process for the $\mathrm{Si}$ microfilter, including (a) $\mathrm{SiO}_{2}$ masking, (b) ICP-RIE, (c) protective coating, (d) back side etching, and (e) cleaning using an HF dip.

were also developed and integrated on the NP sampler head of CANTOR-1. From the market, commercially available grids for transmission electron microscopy (TEM) might be a solution for cavity diameters down to $0.6 \mu \mathrm{m}$. However, those grids were very fragile, leading to difficult mounting and use in our NP sampling system. Therefore, the first stage of microparticle filtration was to use a home-built silicon microfilter with a grid diameter of $2.5 \mu \mathrm{m}$. Its manufacturing processes are illustrated in Fig. 3, which can be described as follows.

1. Silicon dioxide $\left(\mathrm{SiO}_{2}\right)$ was thermally grown on a silicon wafer to be employed as a grid mask by subsequent lithography and HF dips. A second lithography is done to prepare the rim on the front side of the filter.

2. By using ICP cryo-DRIE, the holes with a diameter of $2.5 \mu \mathrm{m}$ were transferred into the silicon wafer.

3. Before structuring the back side of the wafer, its front side had to be firstly protected by a resist layer to prevent mechanical deformation because of harsh contact with the aluminum plate carrier. Moreover, this process flow had been selected to prevent grid artifacts (i.e., a mask undercut that was expected due to the impaired release of the etching-induced heat through a membrane).

4. The backside etching was performed to reduce the membrane thickness down to $\sim 4 \mu \mathrm{m}$. This step is important to determine the ability of the aerosol to pass through the filter channels, because creating too thick a membrane will provide a lower aerosol flow rate, which is a disadvantageous effect for the NP sampling system.

5. After stripping off the photoresist and $\mathrm{SiO}_{2}$ layers, the silicon microfilter had been finally realized.

To check on the utility of the fabricated silicon microfilter shown in Fig. 4a, a preliminary simple test was carried out in the ENP exposure assessment prior to the real-time monitoring of carbon aerosol ENPs (Sigma-Aldrich Chemie $\mathrm{GmbH}$ ). From scanning electron microscope (SEM) investigation, it

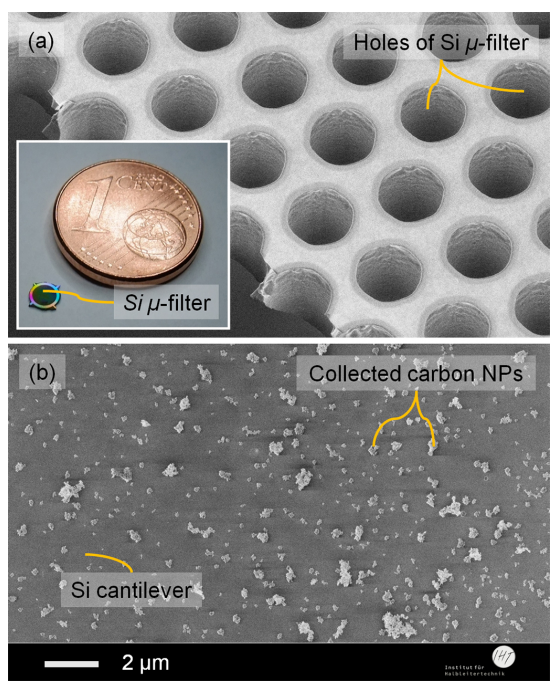

Figure 4. SEM images of the (a) Si microfilter used in CANTOR-1 and (b) the collected carbon ENPs on the cantilever that could pass through the filter holes.

is clearly shown that, by placing the microfilter at the opening inlet of the CANTOR-1 sampler head, the microparticles and their agglomerates $\left(d_{\mathrm{P}}>2.5 \mu \mathrm{m}\right)$ had been rejected. Thus, only the ENPs $\left(d_{\mathrm{P}} \leq 2.5 \mu \mathrm{m}\right)$ could pass through the filter holes and subsequently reach the cantilever surfaces (Fig. 4b).

To further reject the smaller microparticles $\left(d_{\mathrm{P}}>1 \mu \mathrm{m}\right)$, an impactor made of aluminum was also made and placed in between the microfilter and the cantilever sensor, acting as the second large particle filtration stage in the system (Fig. 5a). In other studies, this impaction technology had also been used for various devices and described in detail (Mehdizadeh et al., 2013; Schmid et al., 2013). For our Al impactor, its location is adjustable, which influences the velocity of the aerosol flow. By integrating this impactor, microparticles of diameters in the range of $1 \mu \mathrm{m}<d_{\mathrm{P}} \leq 2.5 \mu \mathrm{m}$ are expected to be impacted, and no longer in the aerosol flow stream. Figure 5b shows as proof microparticles captured on the impactor during a typical particle exposure experiment. Nevertheless, the maximum aerosol velocity in the CANTOR1 system was set to $8-20 \mathrm{~m} \mathrm{~s}^{-1}$ to avoid any disturbances to the operating resonators during the aerosol NP detection. The airflow through the sampler modeled using COMSOL Multiphysics $4.3 \mathrm{~b}$ is depicted in Fig. 5c. The used simulation module is particle tracing.

\subsection{System integration}

Besides the main components (i.e., an NP sampler with its mounted cantilever resonator) and the microparticle filtration stages (i.e., a silicon microfilter and aluminum impactor), there are three other supporting modules (i.e., electronic circuit, power supply, and data acquisition control system), 

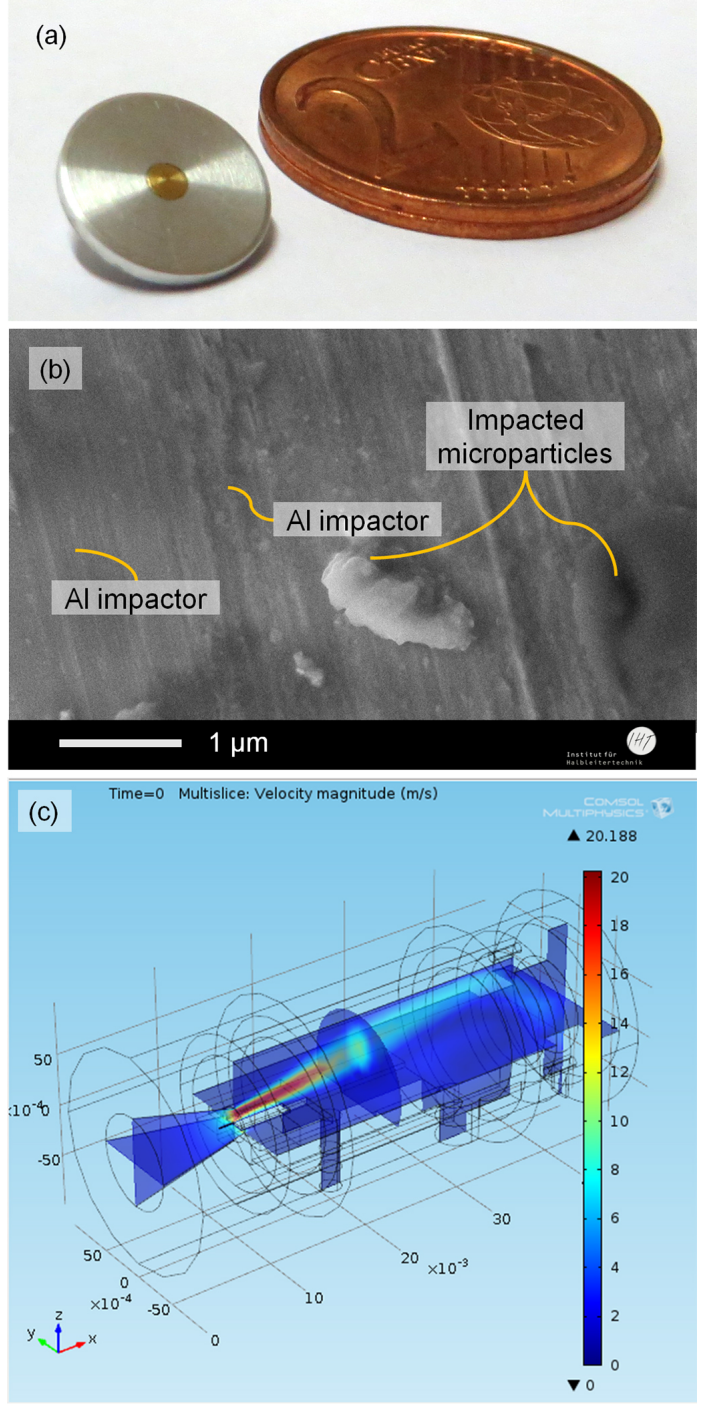

Figure 5. (a) Homebuilt aluminum impactor and (b) impacted microparticles on its surface. (c) 3-D COMSOL simulation result showing the aerosol flow velocity in a color gradient representation ranging from 0 (blue) to $20 \mathrm{~m} \mathrm{~s}^{-1}$ (red) inside the sampler.

which need to be integrated to build a complete CANTOR1 system (Fig. 6). In the electronic circuit module, the frequency tracking system based on PLL and HV modules based on a DC amplifier are realized and involved to track the resonant frequency of the silicon cantilever in real time and amplify the input voltage from $3.5 \mathrm{~V}$ to $0.5 \mathrm{kV}$ in the $\mathrm{NP}$ sampler, respectively (Wasisto et al., 2014). During particle sampling, a negative high voltage of $-0.5 \mathrm{kV}$ is set to one of the cantilever electrodes, which is connected with the bulk contact of the silicon cantilever to generate an electric field over the sensor. Hence, the positively charged and uncharged NPs will be attracted and polarized to the cantilever surfaces by electrophoresis and dielectrophoresis mechanisms, respectively. The detailed mechanism of the airborne NP de-

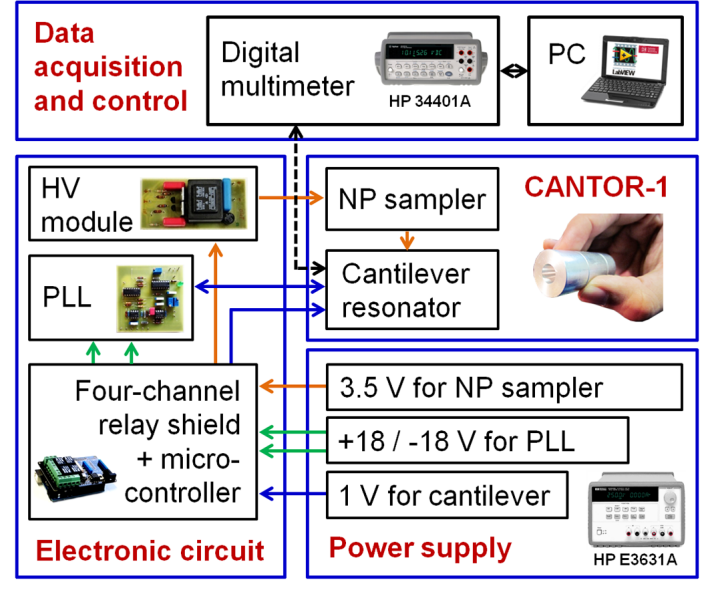

Figure 6. Schematic of the partially integrated CANTOR-1 showing all its components (i.e., a silicon cantilever resonator mounted in an NP sampler, power supply, electronic circuits, and data acquisition control system).

position in an electrostatic sampler had been described by Krinke et al. (2002), who had modeled the NP trajectory as a force balance on a single NP approaching the substrate.

Additionally, a combination of two small electronic components (i.e., an Arduino UNO microcontroller and a Seeedstudio relay shield) was also used to automatically control the periodic switching between NP sampling and resonant frequency measurement phases. The yielded response time of this relay shield was in milliseconds. This fast switching control had overcome the previously raised issue in the software development (i.e., a time delay of about 6-7 s due to the communication overhead between measuring instruments and the notebook computer) (Wasisto et al., 2014). The power sources used in CANTOR-1 originate from the two power supplies (HP 3631A) to supply the different power requirements of the NP sampler (i.e., $3.5 \mathrm{~V}$ ), the cantilever resonator Wheatstone bridge (i.e., $1 \mathrm{~V}$ ), and the PLL circuit (i.e., $\pm 18 \mathrm{~V}$ ).

In general, the operating process of CANTOR-1 can be divided into two phases, which are run alternately. First, the resonant frequency of CANTOR-1 will be tracked with a time resolution of $1 \mathrm{~s}$ under the condition when the PLL integrated with the cantilever sensor is switched on and the NP sampler is switched off by relay. Next, after the PLL integrated with the cantilever sensor has been turned off and the sampler head is subsequently turned on, NP sampling begins to be performed. It should be noted that, during the entire switching, a digital multimeter (HP 34401A) is always kept in reading status to continuously observe the resonant frequency change, which will be further converted into detected NP mass concentration in a LabVIEW-installed PC.

In its present configuration, CANTOR-1 consists of all components for real-time ENP monitoring except those for data acquisition control, logging, and display, for which a 
laptop PC is used. Thus, CANTOR-1 is more than a lab demonstrator, but can also be used as a portable indoor ENP monitor comparable in weight (less than $1 \mathrm{~kg}$, including the PC) with state-of-the-art instrumentation (e.g., an Aerasense nanotracer of $750 \mathrm{~g}$, Buonanno et al., 2014, or a Testo miniDisc of 670 g, Fierz et al., 2011). A fully integrated system, which will include all necessary components in one base unit of a total weight of $<400 \mathrm{~g}$, will be developed as the second version (CANTOR-2).

\section{Detector performance}

\subsection{Test aerosol nanoparticle generation}

After integrating all components into a complete system, CANTOR-1 was then assessed in carbon ENP exposures (Sigma-Aldrich Chemie $\mathrm{GmbH}$ ), which were performed in a test chamber under typical workplace conditions (i.e., $V=$ $1 \mathrm{~m}^{3}, T=23^{\circ} \mathrm{C}, \mathrm{RH}=30 \%$, and $p=1 \mathrm{~atm}$ ), as depicted in Fig. 7a. The generation of stable test aerosols was started by nebulizing a suspension of ENPs in a solution of water/ethanol or water/isobutanol using a constant output atomizer (TSI 3076, TSI Inc.). Inside the atomizer, the incoming compressed air swelled through an orifice forming a highvelocity jet. The liquid solution was then pulled into the atomizing part through a vertical channel and subsequently atomized by the jet. Large droplets were removed by impaction on the barrier opposite to the jet. Meanwhile, the excess liquid was drained at the bottom part of the atomizer to the closed reservoir. As a result, fine spray could leave the atomizer through a channel at the top. By using this technique, the average particle size of the produced aerosol could be varied from 20 to $300 \mathrm{~nm}$.

However, it should be noted that the produced aerosols from the atomizer were still wet. Thus, they had to be dried in a diffusion dryer. In this device, a water trap was incorporated into its inlet to collect coarse water droplets. Moreover, this device consists of two concentric cylinders as the main parts (i.e., inner and outer parts) formed by wire screen and acrylic cylinders, respectively. The space between those two cylinders was filled with a volume of round-shaped silica gel, which acted as a desiccant for having a strong affinity with water molecules and maintaining a dry atmosphere at the tube. During the flowing of the wet aerosol through the inner cylinder, water vapor would diffuse into the silica gel through the wire screen. At the outlet of the dryer, the dried aerosol ENPs can be obtained, which were then flown into a sealed glass chamber (cf. Fig. 7a). To circulate the aerosol inside the chamber (i.e., dynamic condition), a fan was used under a clean air supply of $12 \mathrm{~L} \mathrm{~min}^{-1}$ and put close to one of the inner chamber walls. Furthermore, in situ monitoring of ENP number concentration and size distribution was realized by a fast mobility particle sizer (FMPS, TSI 3091, TSI Inc.) with a time resolution of $1 \mathrm{~s}$ (Fig. 7b). The FMPS worked principally based on the elec-
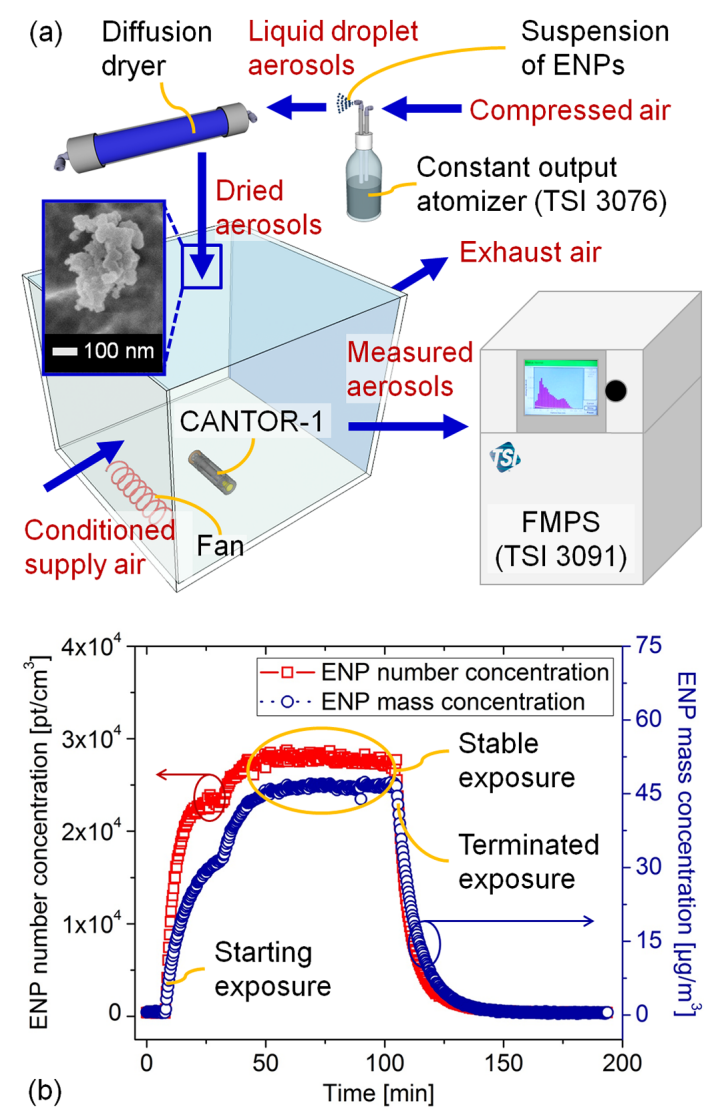

Figure 7. (a) Schematic of the aerosol ENP generation setup in a test chamber, involving an atomizer, a diffusion dryer, a fan, and an FMPS. (b) Typical generated particle number and mass concentrations for carbon airborne ENPs measured by FMPS as a function of time.

trical aerosol spectrometer (EAS) (Tammet et al., 2002). The incoming ENPs, which were firstly charged in a unipolar corona charger, were classified and measured with an array of 22 electrometers, where each channel corresponds to a defined electrical mobility bandwidth. Thus, by knowing the ENP charge levels, the measured currents of the electrometers can be extracted into ENP number size distributions $(6-523 \mathrm{~nm})$. Summing all measured ENP numbers will yield the total concentration of the flowing aerosols inside the chamber. Moreover, by assuming the ENPs are in a spherical shape, their mass concentration can be calculated. The typical stable generated carbon aerosols comprise two size modes of NP diameters of $\sim 20$ and $\sim 120 \mathrm{~nm}$, respectively, at total number concentrations on the order of $10^{4} \mathrm{pt} \mathrm{cm}^{-3}$.

\subsection{Real-time engineered nanoparticle exposure assessments}

The performance of the complete system of CANTOR-1 was evaluated in two real-time ENP exposure tests with different purposes (i.e., a minimum NP sampling investigation and a 
device continuation, respectively). In the first test, the setup of ENP detection was performed according to the specified time (i.e., three cycles comprising 5 min measuring states and varied sampling states $(10,8,6,4,2,1$, and $0.5 \mathrm{~min}$, respectively) for each cycle). CANTOR-1 was automatically switched to the measuring status to track its resonant frequency, whenever the time of the ENP sampling had expired (Fig. 8a).

After experiencing three cycles of repeated exposure tests of carbon aerosol ENPs with a stable concentration of $4.9 \pm 1.2 \times 10^{4} \mathrm{pt} \mathrm{cm}^{-3}$ in a sequence of periodic states (i.e., consecutive sampling and measuring), a real-time behavior of CANTOR-1 can then be observed. The increasing mass loading of the deposited ENPs had caused a linear drop in cantilever resonant frequency during a total sampling time of $94.5 \mathrm{~min}$ (Fig. 8b). From the analyzed results, the collected ENP mass amounted to $\Delta m_{\mathrm{ENP}}=2 \times m_{\text {cant }} \times \Delta f_{\mathrm{ENP}} / f_{\text {cant }}=$ $26.88 \mathrm{ng}$, which corresponded to a frequency shift of $\Delta f_{\mathrm{ENP}}=-3.95 \mathrm{~Hz}$. Thus, a mass sensitivity of CANTOR1 of $S_{\mathrm{ENP}}=\Delta f_{\mathrm{ENP}} / \Delta m_{\mathrm{ENP}}=-0.15 \mathrm{~Hz} \mathrm{ng}^{-1}$ could be calculated under this condition. Furthermore, from this test, it was shown that there was a necessary waiting time (i.e., $\sim 1-2$ min) of the resonant frequency measurement before CANTOR-1 could operate under a stable condition, which was related to the PLL capturing time. Moreover, the ability of CANTOR-1 to detect the airborne ENP within only $1 \mathrm{~min}$ sampling had been confirmed, although some unstable data points were also present, which could be attributed to an unwanted lift-off of already deposited ENP fractions.

Next, differentiating from the first online test where the measurement was done in only a day, the second exposure assessment of CANTOR-1 was performed on two subsequent days to investigate the continuation of device use. Moreover, even though, in the first test of online measurement, CANTOR-1 could already detect carbon ENPs with only $1 \mathrm{~min}$ sampling time resolution, in the second test, CANTOR-1 was set at 5 min sampling time and 3 min measuring time to better interpret the data (i.e., a sufficient frequency shift). Thus, in total, CANTOR-1 will need $8 \mathrm{~min}$ to obtain a single data point of the ENP monitoring. This value is well below the $10-15$ min period recommended for shortterm aerosol exposure measurement (Duarte et al., 2014).

For the first day of the second online test, CANTOR-1 was operated only to measure the collected ENP mass in a stable carbon aerosol concentration of $2.1 \pm 0.3 \times 10^{4} \mathrm{pt} \mathrm{cm}^{-3}$ (i.e., $33 \pm 7 \mu \mathrm{g} \mathrm{m}^{-3}$ ). Therefore, the concentration inside the chamber after the ENP exposure termination (i.e., aerosol evacuating process) was not recorded. As expected, the similar effect to that obtained from the first online test was also clearly seen in this experiment (Fig. 9a). The linear decrease in the CANTOR-1 resonant frequency (i.e., $-0.07 \pm 0.02 \mathrm{~Hz} \mathrm{~min}^{-1}$ ) was proportional to the increase in the collected ENP mass (i.e., $0.46 \pm 0.11 \mathrm{ng} \mathrm{min}^{-1}$ ), with an ENP sampling efficiency of $2.1 \pm 0.5 \%$. In the last period of the measurement, the resonant frequency of CANTOR-1 be-
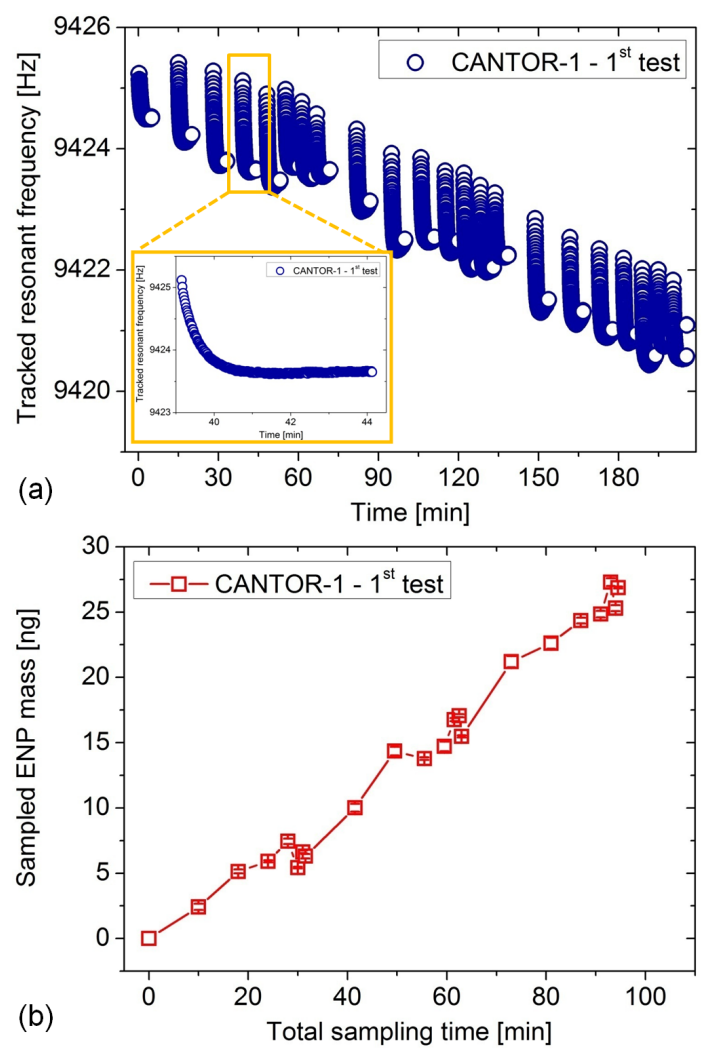

Figure 8. (a) The tracked resonant frequency and (b) its corresponding collected ENP mass monitored with CANTOR-1 during the first test of carbon ENP sampling.

fore being switched off was $9422.03 \pm 0.04 \mathrm{~Hz}$, which was also identical for the initial value on the next measurement day.

On the second day of the second online test, CANTOR-1 was kept operational to measure the ENP mass concentration from the early beginning of the exposure up to the end of the chamber evacuation of ENPs. However, at a point where the proof of stable ENP mass concentration has been sufficient, the flowing of the dispersed aerosols to the chamber was stopped. This action was used to validate the CANTOR1 performance in terms of its sensitivity to the transitions of the different ENP concentrations from low to high exposure levels and in the opposite way. It can be obviously seen from Fig. 9b that the CANTOR-1 resonant frequency gradually decreased as the aerosol ENPs started to be injected into the chamber. This frequency reduction became linear (i.e., $-0.05 \pm 0.03 \mathrm{~Hz} \mathrm{~min}^{-1}$, corresponding to a collected mass of $0.34 \pm 0.09 \mathrm{ng} \mathrm{min}^{-1}$ with an ENP sampling efficiency of $1.1 \pm 0.6 \%$ ) after the aerosols reached their stable condition of $2.7 \pm 0.2 \times 10^{4} \mathrm{pt} \mathrm{cm}^{-3}$ (i.e., $46 \pm 1 \mu \mathrm{g} \mathrm{m}^{-3}$ ). However, after exposure termination, the CANTOR-1 resonant frequency did not degrade into lower values anymore. Instead, it stayed at almost constant values of $9418.93 \pm 0.13 \mathrm{~Hz}$, meaning that there were no ENPs being sampled on the cantilever surfaces. 

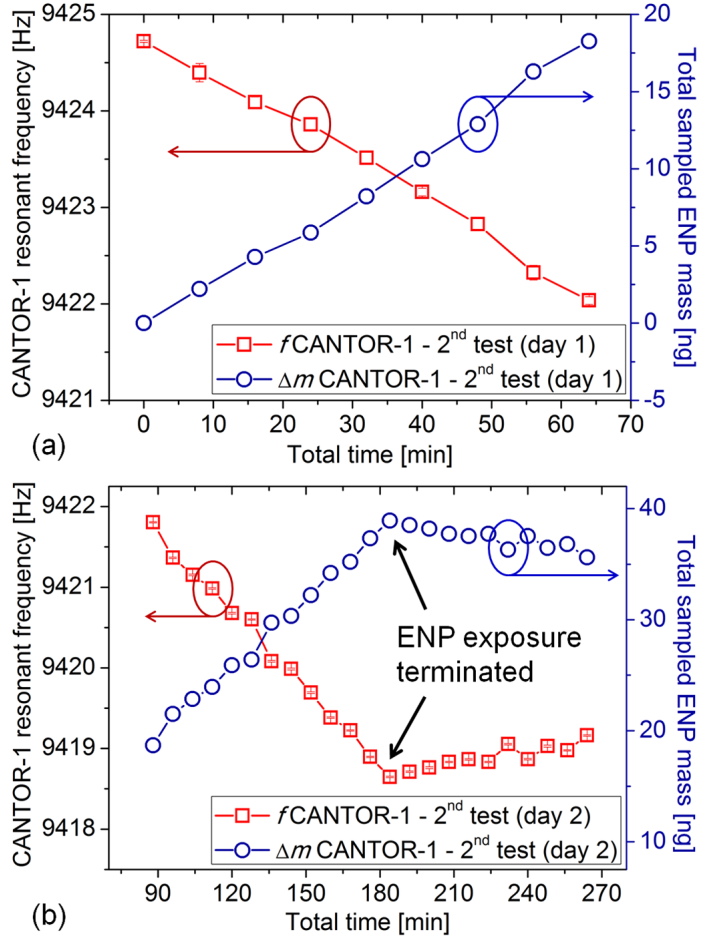

Figure 9. The tracked resonant frequency of CANTOR-1 during the second test of carbon ENP sampling and its corresponding collected ENP mass for (a) day 1 and (b) day 2. On day 2, CANTOR-1 was kept operational after ENP exposure termination.

There was even a slight decrease in total sampled ENP mass. It could be a condition where the already deposited ENPs or their agglomerates were being lifted off again as the cantilever sensor of CANTOR-1 kept vibrating.

\subsection{Detector calibration}

Although CANTOR-1 had already exhibited good performance in the two online ENP mass monitoring tests as a microbalance, it still became a necessity to calibrate this homebuilt device with a standard NP monitoring instrument in well-defined aerosols to recalculate the measured data in the standard unit for aerosol mass concentration (i.e., $\mu \mathrm{g} \mathrm{m}^{-3}$ ). Thus, during measurement on the second day of the second online test, the results obtained by CANTOR-1 are also calibrated with FMPS (TSI 3091) (cf. Figs. 7b and 9b). The calibrated ENP mass concentration $\left(C_{m_{-} \text {CANTOR-1 }}\right)$ in $\mu \mathrm{g} \mathrm{m}^{-3}$ can be calculated using

$$
\begin{aligned}
C_{m \_ \text {CANTOR-1 }}\left(\mu \mathrm{g} \mathrm{m}^{-3}\right) & =\mathrm{CF}(T, \mathrm{RH}, p) \\
& \times \frac{\Delta f}{\Delta t}\left(\mathrm{~Hz} \mathrm{~min}^{-1}\right),
\end{aligned}
$$

where $\mathrm{CF}$ is the calibration factor in terms of the interferences from temperature, relative humidity, and pressure.

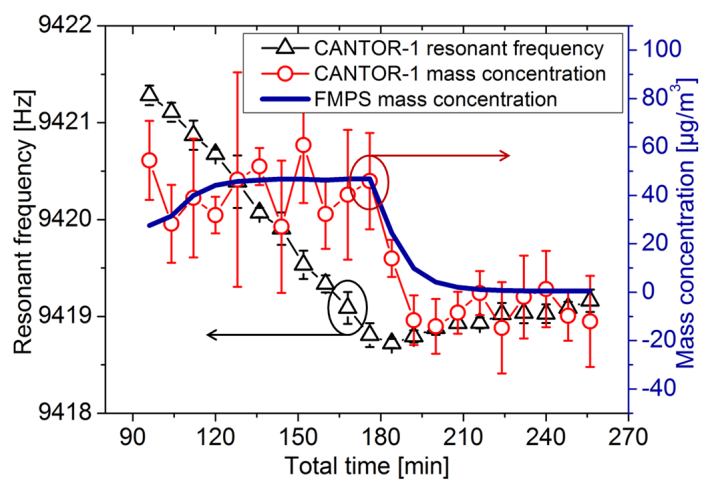

Figure 10. The tracked resonant frequency and its corresponding ENP mass concentration calibrated towards FMPS (TSI 3091) monitored with CANTOR-1 during the second day test of real-time carbon ENP sampling.

Meanwhile, $\Delta f / \Delta t$ is the resonant frequency shift per time unit of CANTOR-1 (i.e., $\mathrm{Hz} \mathrm{min}^{-1}$ ). However, prior to the determination of $\mathrm{CF}$, the ENP number concentration measured by FMPS (i.e., $C_{n_{-} \text {FMPS }}$ ) must first be converted into ENP mass concentration (i.e., $C_{m_{-} \text {FMPS }}$ ) by assuming that the size-distributed particles are in a spherical shape. The CF value (i.e., $\left.\mu \mathrm{g} \min \left(\mathrm{m}^{3} \mathrm{~Hz}\right)^{-1}\right)$, in this case, is given by

$\mathrm{CF}=\frac{C_{m_{-} \text {FMPS_avg }}}{X_{\Delta f / \Delta t \_ \text {avg }}}\left(\mu \mathrm{g} \min \left(\mathrm{m}^{3} \mathrm{~Hz}\right)^{-1}\right)$,

where $C_{m \_F M P S \_a v g}$ and $X_{\Delta f / \Delta t \_a v g}$ are the averaged value of $C_{m}$ FMPS under stable conditions and its corresponding mean value of the CANTOR resonant frequency shift per time unit, respectively.

Figure 10 shows the calibrated ENP mass concentrations measured by CANTOR-1 in comparison with those measured by FMPS. In this case, the CF used for CANTOR-1 was $815 \mu \mathrm{g} \min \left(\mathrm{m}^{3} \mathrm{~Hz}\right)^{-1}$. During $1 \mathrm{~h}$ assessment of a stable carbon ENP mass concentration of $46 \pm 1 \mu \mathrm{g} \mathrm{m}{ }^{-3}$ measured by FMPS, the precision of the calibrated ENP concentrations measured by CANTOR-1 was found to be $<55 \%$, which was taken as the deviation of each measured data point. Moreover, by multiplying the minimum frequency shifts that can be resolved with $\mathrm{CF}$, a limit of detection (LOD) of $<25 \mu \mathrm{g} \mathrm{m}^{-3}$ was obtained for this detector. Regardless of some drawbacks of CANTOR-1 (i.e., time-consuming sensor preparation and a low ENP sampling efficiency of $1.07 \%$ ), the overall experimental results have verified the good sensitivity and working status of CANTOR-1, because in the real workplace, this detector aims to be used as a first alert for informing the workers in regards to the danger caused by the unexpected excessive concentration of the ENPs in air that can occur suddenly during their working shifts. 

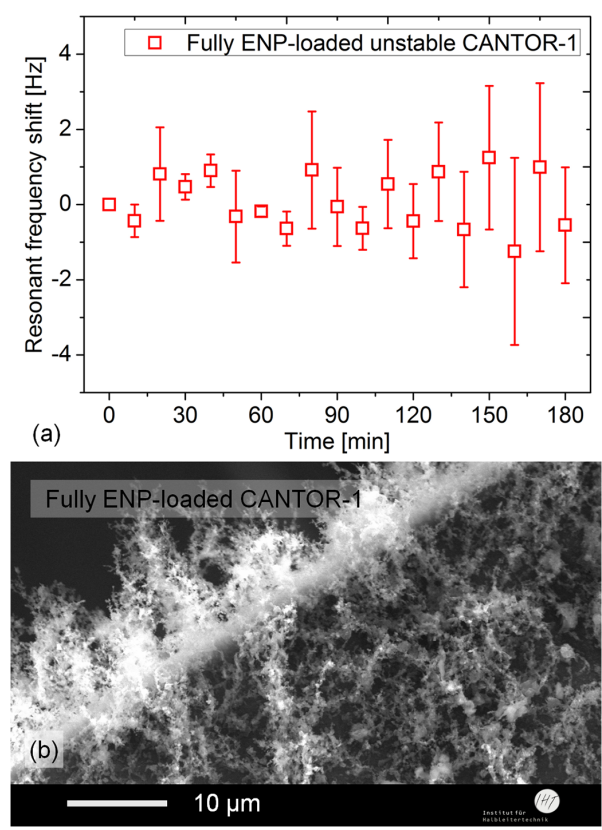

Figure 11. (a) Typical unstable resonant frequency signal and (b) surface condition of a fully ENP-loaded CANTOR-1.

\subsection{Detector robustness}

To test the robustness of CANTOR-1 in terms of its operating lifetime, long-term use during a carbon ENP exposition of $\sim 1-7 \times 10^{4} \mathrm{pt} \mathrm{cm}^{-3}$ was performed with a recycled silicon piezoresistive cantilever integrated into CANTOR-1 within 7 workdays. It was found that the signal of CANTOR1 started to get unstable only after $43.5 \mathrm{~h}$ of ENP sampling as compared to the normal device performance (Fig. 11a). Thus, $\sim 40 \mathrm{~h}$ of continuous operations can be considered the lifetime of CANTOR-1 under typical mass concentrations in the ambience at workplaces of $20-120 \mu \mathrm{g} \mathrm{m}^{-3}$. For the corresponding frequency shift and collected ENPs, we found $-79.15 \mathrm{~Hz}$ and $0.54 \mu \mathrm{g}$, respectively. Using the cantilever mass of $m_{\text {cant }} \approx 32 \mu \mathrm{g}$, we conclude that the operating life of CANTOR-1 ends when the mass of deposited ENPs reaches a value of $\sim 2 \%$ of the cantilever weight. This fact was supported by SEM, showing the surface of the cantilever heavily loaded by carbon ENPs (Fig. 11b). Almost all of the deposited ENPs exhibit an agglomerate shape. Thus, the unstable signal of CANTOR-1 might be explained by a loss of mass by detaching of large ENP agglomerates of highenough inertia.

To regenerate the fully ENP-loaded cantilever, a relatively simple but efficient wet cleaning method was demonstrated using an ultrasonic cleaner with acetone solution or deionized (DI) water inside. This ultrasonic cleaning technique had been implemented in several heavily used cantilever sensors with varied geometries (i.e., down to nanoscaled devices) and exposure levels, which had always obtained high cleaning efficiencies up to $\sim 99 \%$ (Wasisto et al., 2013c, d).

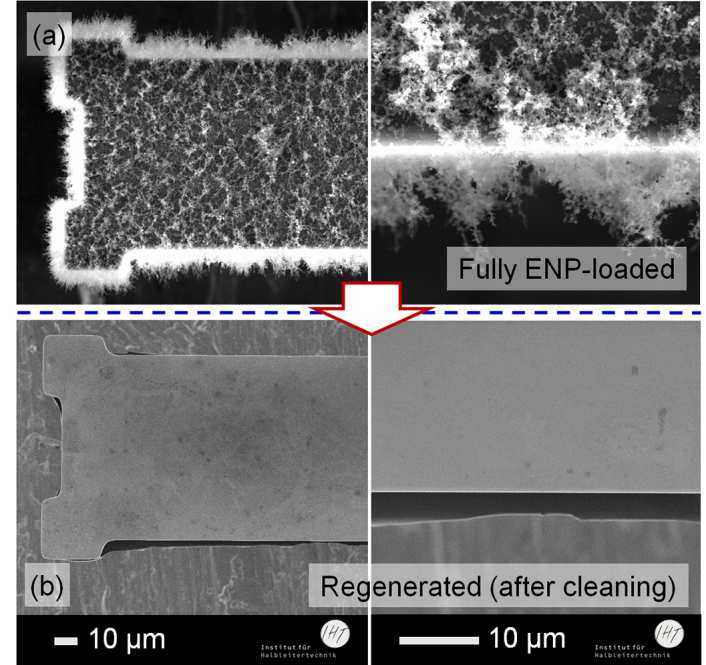

Figure 12. The surface conditions of fully ENP-loaded CANTOR1 (a) before and (b) after ultrasonic cleaning.

During the high pressure stage of the cleaning process using a Bandelin Sonorex Ultrasonic bath TK $52(f=35 \mathrm{kHz})$, the formed bubbles imploded, releasing enormous amounts of energy attacking every surface; hence, after $0.5-2 \mathrm{~min}$, the deposited ENPs were almost completely detached from the cantilever surface. Figure 12a and b depict the surface conditions of the heavily ENP-loaded cantilever of CANTOR-1 before and after the ultrasonic wet cleaning process, respectively. Nevertheless, considering their potentially low fabrication cost (i.e., fabricated using bulk silicon instead of SOI wafers), the cantilevers may also be considered a disposable component. Since spring-loaded contact pins are used, wirebonding is not required for electrical contact to the cantilever die. Thus, it can be replaced easily with either a new or a regenerated one after some period of the ENP sampling.

\subsection{Detector comparison}

To compare the developed CANTOR-1 with the other up-todate MEMS-/NEMS-based particle detectors worldwide, Table 1 has summarized their important data (i.e., measurement principle, LOD, particle sampling time, and notes). From this comparison, it is clearly shown that only CANTOR-1 uses the electrophoretic sampling method. The other prototypes from the University of Denver (DU, USA; Hajjam et al., 2011), the Berkeley Sensor and Actuator Center (BSAC, USA; Paprotny et al., 2013), the Technical University of Denmark (DTU, Denmark; Schmid et al., 2013), and McGill University (Canada; Morris et al., 2014), employ impaction and thermophoretic methods. The slowest response is exhibited by quartz crystal microbalance (QCM) from McGill University, with a 30 min sampling time. Moreover, the detected targets of the devices from McGill University, DU, and BSAC were large particles (i.e., microparticles). They 
Table 1. Comparison between the developed partially integrated CANTOR-1 and the other currently researched micro/nanomechanical particle detectors.

\begin{tabular}{|c|c|c|c|}
\hline Developer, reference & Measurement principle & $\begin{array}{l}\text { LOD and } \\
\text { particle } \\
\text { sampling time }\end{array}$ & Notes \\
\hline $\begin{array}{l}\text { DU, USA (Hajjam et al., } \\
\text { 2011) }\end{array}$ & $\begin{array}{l}\text { Direct deposition in a partial } \\
\text { vacuum and thermally actuated } \\
\text { silicon resonator }\end{array}$ & $\begin{array}{l}3 \mu \mathrm{g} \mathrm{m}^{-3} \\
10 \mathrm{~s}\end{array}$ & $\begin{array}{l}\text { Partial vacuum, microscope and } \\
\text { large pump needed, microparticle } \\
\text { detection, no sensor recycling pro- } \\
\text { cess }\end{array}$ \\
\hline $\begin{array}{l}\text { BSAC, LBNL, and EPA, } \\
\text { USA (Paprotny et al., 2013) }\end{array}$ & $\begin{array}{l}\text { Thermophorectic precipitator } \\
\text { and film bulk acoustic resonator } \\
\text { (FBAR) }\end{array}$ & $\begin{array}{l}2 \mu \mathrm{g} \mathrm{m}^{-3} \\
10 \mathrm{~min} \\
10 \mu \mathrm{g} \mathrm{m}^{-3} \\
4 \mathrm{~min}\end{array}$ & $\begin{array}{l}\text { High particle loss, fan stack } \\
\text { needed, non-integrated electronic } \\
\text { circuitries, no sensor recycling } \\
\text { process }\end{array}$ \\
\hline $\begin{array}{l}\text { DTU, Denmark (Schmid et } \\
\text { al., 2013) }\end{array}$ & $\begin{array}{l}\text { Inertial impactor and nanome- } \\
\text { chanical resonant filter fiber }\end{array}$ & $\begin{array}{l}<1 \mu \mathrm{g} \mathrm{m}^{-3} \\
1 \mathrm{~s}\end{array}$ & $\begin{array}{l}\text { High particle loss, external pump } \\
\text { needed, bulky measurement tools } \\
\text { required (i.e., lock-in amplifier and } \\
\text { laser Doppler vibrometer), no sen- } \\
\text { sor recycling process }\end{array}$ \\
\hline $\begin{array}{l}\text { McGill University, Canada } \\
\text { (Morris et al., 2014) }\end{array}$ & $\begin{array}{l}\text { Impactor and quartz crystal mi- } \\
\text { crobalance (QCM) }\end{array}$ & $\begin{array}{l}5.2 \mu \mathrm{g} \mathrm{m}^{-3} \\
30 \mathrm{~min}\end{array}$ & $\begin{array}{l}\text { Bulky measurement tools and vac- } \\
\text { uum pump needed, microparticle } \\
\text { detection, no sensor recycling pro- } \\
\text { cess }\end{array}$ \\
\hline $\begin{array}{l}\text { IHT TU Braunschweig and } \\
\text { Fraunhofer WKI, Germany } \\
\text { (this work) }\end{array}$ & $\begin{array}{l}\text { Electrophoretic precipitator and } \\
\text { self-sensing silicon cantilever } \\
\text { resonator actuated with a piezo- } \\
\text { electric actuator (CANTOR-1) }\end{array}$ & $\begin{array}{l}25 \mu \mathrm{g} \mathrm{m}^{-3} \\
1-5 \mathrm{~min}\end{array}$ & $\begin{array}{l}\text { Partially integrated, miniaturized } \\
\text { sampler, two-stage microparticle } \\
\text { filtrations, sensor recycling process } \\
\text { demonstrated, sensor calibrated, } \\
\text { digital multimeter and power } \\
\text { supply required }\end{array}$ \\
\hline
\end{tabular}

do not have any filtering systems for smaller particles (i.e., NPs). Some of them even had to be operated with a large vacuum pump and the optical method to yield the aerosol flows and read out the signal, which are not applicable for development of low-cost wearable sensor systems. Although most of them provide slightly better LODs than CANTOR1 , they have not demonstrated a method to remove the deposited particles and recycle their sensors. Furthermore, for the nanowire device from DTU, it is expected that the sensor will be fully loaded by NPs within only a few minutes. As an improvement to CANTOR-1, its next generation (i.e., CANTOR-2) will be developed as a fully integrated system, where all sensing components and their supporting electronics will be miniaturized and packed into handy-format housing. Hence, it can be worn easily by workers in nanotechnology industries during their working shifts. According to the paradigm shift reported for air pollution monitoring towards lower-cost, easy-to-use, portable, and direct-reading sensors, there is further commercial potential for ENP monitoring to be expected in the near future (Kumar et al., 2015; Snyder et al., 2013).

\section{Conclusions}

The first generation of a low-cost portable cantilever-based airborne nanoparticle detector (i.e., CANTOR-1) has been developed as a partially integrated direct-reading device, assessed with carbon aerosol engineered nanoparticles (ENPs) under typical workplace conditions, and calibrated towards a standard ENP monitoring instrument of a fast mobility particle sizer (FMPS, TSI 3091) with a measurement precision of $<55 \%$ and a limit of detection (LOD) of $<25 \mu \mathrm{g} \mathrm{m}^{-3}$. The two-stage filtrations have also been tested, leading to a rejection of micro-sized particles $\left(d_{\mathrm{P}}>1 \mu \mathrm{m}\right)$. The cantilever module of CANTOR-1 could also be regenerated using the ultrasonic cleaning method after being fully loaded by ENPs in a long-term ENP sampling. Further work is needed to improve the sensing performance and to pack all components into a fully wearable handy NP detector. 


\section{Biographies}

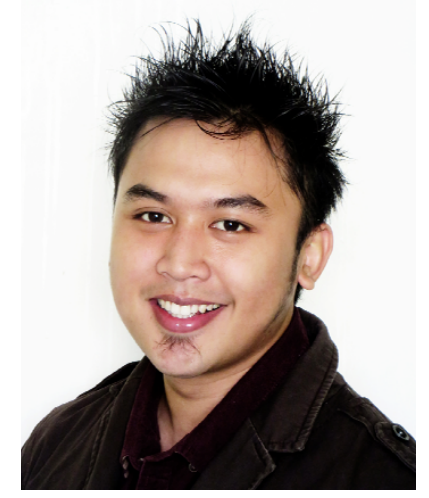

Hutomo Suryo Wasisto received the Bachelor of Engineering degree in electrical engineering from Gadjah Mada University, Indonesia, the Master of Engineering degree in semiconductor engineering from Asia University, Taiwan, and the Doktor-Ingenieur (Dr.-Ing.) degree in electrical engineering with the Summa Cum Laude honor from the Technische Universität Braunschweig, Germany, in 2008, 2010, and 2014, respectively. Currently, he is working as a senior research scientist at the Institute of Semiconductor Technology (IHT), TU Braunschweig and the Laboratory for Emerging Nanometrology (LENA), Germany, where his main interests are in the fields of micro-/nano-electromechanical systems (MEMS/NEMS)-based silicon cantilever technology for personal aerosol nanoparticle sensing devices and high-speed measurements of surfaces of microstructures with high aspect ratios. He has published more than 40 papers in international scientific journals and conference proceedings. He has also been the recipient of the best paper award and the second best young scientist poster award at the 8th IEEE International Conference on Nano/Micro Engineered and Molecular Systems (IEEE NEMS 2013) in Suzhou, China, and the 26th European Conference on Solid-State Transducers (Eurosensors 2012) in Krakow, Poland, respectively. In 2014, he received the Walter-Kertz-Studienpreis (Walter Kertz Study Award) for his excellent doctoral dissertation and achievements of scientific studies at the interface between physics, electrical engineering, and information technology from the TU Braunschweig, Germany.

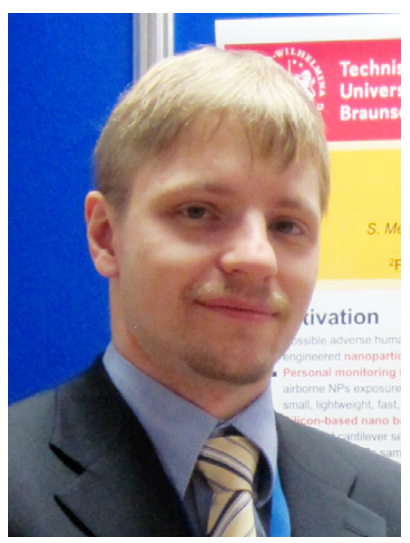

Stephan Merzsch received the Diplomingenieur (Dipl.-Ing.) degree in electrical engineering from the Braunschweig University of Technology, Braunschweig, Germany, in 2008. Since 2008, he has been a PhD student at the Institute of Semiconductor Technology (IHT), TU Braunschweig, Germany. His main interests are semiconductor sensors, microsystems technology, and nanoimprint techniques for airborne nanoparticle sensors. Currently, he is working for Infineon Technologies AG, Munich, Germany.

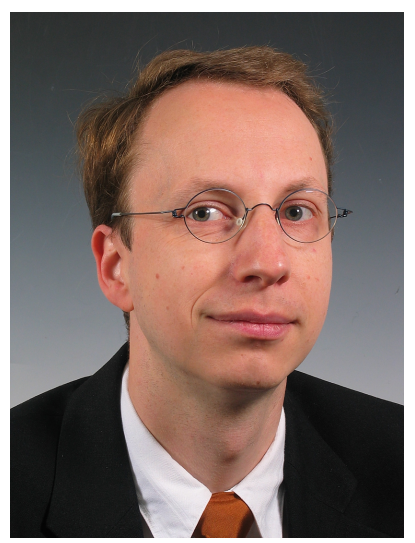

Erik Uhde received his diploma in chemistry (1994) and later his PhD (physical chemistry, 1998) from the Braunschweig University of Technology, Germany. In 1995, he started as a project manager at Fraunhofer WKI. Since 2001, he has been the deputy head of the Department of Material Analysis and Indoor Chemistry, which specializes in the characterization of gaseous and solid air pollutants indoors. $\mathrm{He}$ is a member of national and international standardization groups in the field of indoor air quality. 


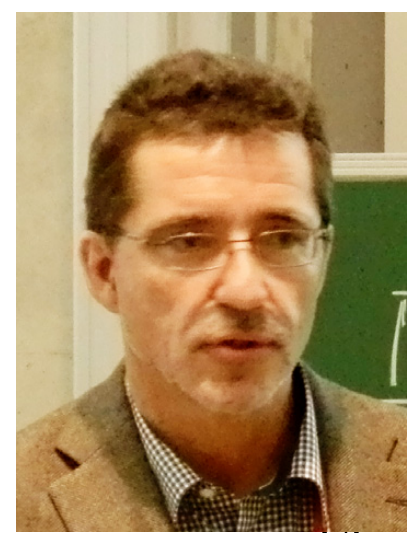

Andreas Waag received his diploma as well as PhD degree in physics from the University of Würzburg, Germany, in 1985 and 1990. In 1996, he got the Gaede Award of the German Vacuum Society for the development of novel II-VI materials for blue-green laser diodes. Since 2003, he has been a full professor at TU Braunschweig, University of Technology, and head of the Institute of Semiconductor Technology (IHT), with activities in the field of oxides and nitrides for optoelectronics, as well as sensor and joining technology.

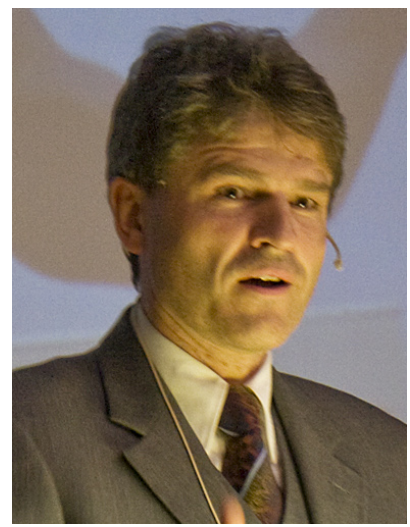

Erwin Peiner received the Diplom-Physiker and $\mathrm{PhD}$ degrees from the University of Bonn, Germany, in 1985 and 1988, respectively. In 2000 he received the venia legendi for semiconductor technology from the Faculty of Mechanical and Electrical Engineering of the TU Braunschweig. Currently, he is the leader of the semiconductor sensors and metrology group at the Institute of Semiconductor Technology (IHT) of the TU Braunschweig. He has published more than 250 papers in international journals and conference proceedings. He has been the project coordinator of the collaborative NanoExpo project funded by the German Federal Ministry of Education and Research (BMBF).
Acknowledgements. The authors would like to thank I. Kirsch, Q. Zhang, K. Huang, Z. Wang, W. Wu, J. Arens, D. Rümmler, and K.-H. Lachmund for their valuable technical support. This work was performed in the NanoExpo collaborative project funded by the German Federal Ministry of Education and Research (BMBF) within the NanoCare cluster under number 03X0098A/B.

Edited by: T. Fröhlich

Reviewed by: three anonymous referees

\section{References}

Balbus, J. M., Florini, K., Denison, R. A., and Walsh, S. A.: Protecting workers and the environment: An environmental NGO's perspective on nanotechnology, J. Nanopart. Res., 9, 11-22, doi:10.1007/s11051-006-9173-7, 2007.

Bekker, C., Brouwer, D. H., Tielemans, E., and Pronk, A.: Industrial production and professional application of manufactured nanomaterials-enabled end products in Dutch industries: Potential for exposure, Ann. Occup. Hyg., 57, 314-327, doi:10.1093/annhyg/mes072, 2013.

Brouwer, D.: Exposure to manufactured nanoparticles in different workplaces, Toxicology, 269, 120-127, doi:10.1016/j.tox.2009.11.017, 2010.

Buonanno, G., Jayaratne, R. E., Morawska, L., and Stabile, L.: Metrological performances of a diffusion charger particle counter for personal monitoring, Aerosol Air Qual. Res., 14, 156-167, doi:10.4209/aaqr.2013.05.0152, 2014.

Cho, C.-H., Jaeger, R. C., and Suhling, J. C.: Characterization of the temperature dependence of the piezoresistive coefficients of silicon from $-150^{\circ} \mathrm{C}$ to $+125^{\circ} \mathrm{C}$, IEEE Sens. J., 8, 1455-1468, doi:10.1109/JSEN.2008.923575, 2008.

Duarte, K., Justino, C. I. L., Freitas, A. C., Duarte, A. C., and Rocha-Santos, T. A. P.: Direct-reading methods for analysis of volatile organic compounds and nanoparticles in workplace air, Trend Anal. Chem., 53, 21-32, doi:10.1016/j.trac.2013.08.008, 2014.

Fierz, M., Houle, C., Steigmeier, P., and Burtscher, H.: Design, calibration, and field performance of a miniature diffusion size classifier, Aerosol Sci. Tech., 45, 1-10, doi:10.1080/02786826.2010.516283, 2011.

Hajjam, A., Wilson, J. C., and Pourkamali, S.: Individual airborne particle mass measurement using high-frequency micromechanical resonators, IEEE Sens. J., 11, 2883-2890, doi:10.1109/JSEN.2011.2147301, 2011.

Hullman, A.: Measuring and assessing the development of nanotechnology, Scientometrics, 70, 739-758, doi:10.1007/s11192007-0310-6, 2007.

Krinke, T. J., Deppert, K., Magnusson, M. H., Schmidt, F., and Fissan, H.: Microscopic aspects of the deposition of nanoparticles from the gas phase, Aerosol Sci., 33, 1341-1359, doi:10.1016/S0021-8502(02)00074-5, 2002.

Kumar, P., Morawska, L., Martani, C., Biskos, G., Neophytou, M., Di Sabatino, S., Bell, M., Norford, L., and Britter, R.: The rise of low-cost sensing for managing air pollution in cities, Environ. Int., 75, 199-205, doi:10.1016/j.envint.2014.11.019, 2015.

Maynard, A. D.: Nanotechnology the next big thing, or much ado about nothing?, Ann. Occup. Hyg., 51, 1-12, doi:10.1093/annhyg/mel071, 2007. 
Mehdizadeh, E., Kumar, V., Pourkamali, S., Gonzales, J., and Abdolvand, R.: A two-stage aerosol impactor with embedded MEMS resonant mass balances for particulate size segregation and mass concentration monitoring, Proceedings of IEEE SENSORS 2013, Baltimore, USA, 1-4, doi:10.1109/ICSENS.2013.6688317, 2013.

Morris, D. R. P., Fatisson, J., Olsson, A. L. J., Tufenkji, N., and Ferro, A. R.: Real-time monitoring of airborne cat allergen using a QCM-based immunosensor, Sensor. Actuat. B-Chem., 190, 851-857, doi:10.1016/j.snb.2013.09.061, 2014.

Paprotny, I., Doering, F., Solomon, P. A., White, R. M., and Gundel, L. A.: Microfabricated air-microfluidic sensor for personal monitoring of airborne particulate matter: Design, fabrication, and experimental results, Sensor. Actuat. A-Phys., 201, 506-516, doi:10.1016/j.sna.2012.12.026, 2013.

Peiner, E., Balke, M., and Doering, L.: Slender tactile sensor for contour and roughness measurements within deep and narrow holes, IEEE Sens. J., 8, 1960-1967, doi:10.1109/JSEN.2008.2006701, 2008.

Schmid, S., Kurek, M., Adolphsen, J. Q., and Boisen, A.: Real-time single airborne nanoparticle detection with nanomechanical resonant filter-fiber, Scientific Reports, 3, 1288, doi:10.1038/srep01288, 2013.

Snyder, E. G., Watkins, T. H., Solomon, P. A., Thoma, E. D., Williams, R. W., Hagler, G. S. W., Shelow, D., Hindin, D. A., Kilaru, V. J., and Preuss, P. W.: The changing paradigm of air pollution monitoring, Environ. Sci. Technol., 47, 11369-11377, doi:10.1021/es4022602, 2013.

Sökmen, Ü., Stranz, A., Waag, A., Ababneh, A., Seidel, H., Schmid, U., and Peiner, E.: Evaluation of resonating Si cantilevers sputter-deposited with AlN piezoelectric thin films for mass sensing applications, J. Micromech. Microeng., 20, 064007, doi:10.1088/0960-1317/20/6/064007, 2010.
Tammet, H., Mirme, A., and Tamm, E.: Electrical aerosol spectrometer of Tartu University, Atmos. Res., 62, 315-324, doi:10.1016/S0169-8095(02)00017-0, 2002.

Wasisto, H. S., Merzsch, S., Waag, A., Uhde, E., Salthammer, T., and Peiner, E.: Portable cantilever-based airborne nanoparticle detector, Sensor. Actuat. B-Chem., 187, 118-127, doi:10.1016/j.snb.2012.09.074, 2013a.

Wasisto, H. S., Merzsch, S., Waag, A., Uhde, E., Salthammer, T., and Peiner, E.: Evaluation of photoresist-based nanoparticle removal method for recycling silicon cantilever mass sensors, Sensor. Actuat. A-Phys., 202, 90-99, doi:10.1016/j.sna.2012.12.016, $2013 b$.

Wasisto, H. S., Merzsch, S., Stranz, A., Waag, A., Uhde, E., Salthammer, T., and Peiner, E.: Silicon resonant nanopillar sensors for airborne titanium dioxide engineered nanoparticle mass detection, Sensor. Actuat. B-Chem., 189, 146-156, doi:10.1016/j.snb.2013.02.053, 2013c.

Wasisto, H. S., Merzsch, S., Stranz, A., Waag, A., Uhde, E., Salthammer, T., and Peiner, E.: Femtogram aerosol nanoparticle mass sensing utilising vertical silicon nanowire resonators, IET Micro \& Nano Letters, 8, 554-558, doi:10.1049/mnl.2013.0208, 2013d.

Wasisto, H. S., Zhang, Q., Merzsch, S., Waag, A., and Peiner, E.: A phase-locked loop frequency tracking system for portable microelectromechanical piezoresistive cantilever mass sensors, $\mathrm{Mi}$ crosyst. Technol., 20 559-569, doi:10.1007/s00542-013-1991-9, 2014. 